\title{
Differences in Levodopa Response for Progressive and Non-Progressive Micrographia in Parkinson's Disease
}

\author{
Poonam Zham 1,2, Sridhar A. Poosapadi ${ }^{1,3+}$, Peter Kempster ${ }^{4,5}$, Sanjay Raghav ${ }^{1,5}$, \\ Kanae J. Nagao ${ }^{5}$, Kitty Wong ${ }^{5}$ and Dinesh Kumar ${ }^{1 *}$ \\ ${ }^{1}$ School of Engineering, RMIT University, Melbourne, VIC, Australia, ${ }^{2}$ IBM Research Australia, Southbank, VIC, Australia, \\ ${ }^{3}$ Center for Human Movement Research and Analysis, Department of Electronics and Instrumentation, SRM Institute of \\ Science and Technology, Chennai, India, ${ }^{4}$ Department of Medicine, Monash University, Clayton, VIC, Australia, \\ ${ }^{5}$ Neurosciences Department, Monash Medical Centre, Melbourne, VIC, Australia
}

\section{OPEN ACCESS}

Edited by:

Salvatore Galati,

Neurocenter of Southern

Switzerland, Switzerland

Reviewed by:

Matteo Bologna,

Sapienza University of Rome, Italy

Carlo Alberto Artusi,

University of Turin, Italy

*Correspondence:

Dinesh Kumar

dinesh@rmit.edu.au

tPresent address:

Sridhar A. Poosapadi,

Center for Human Movement

Research and Analysis, Department of Electronics and Instrumentation, SRM Institute of Science and Technology,

Chennai, India

Specialty section: This article was submitted to Movement Disorders, a section of the journal Frontiers in Neurology

Received: 07 February 2021 Accepted: 09 April 2021

Published: 11 May 2021

Citation:

Zham P, Poosapadi SA, Kempster P, Raghav S, Nagao KJ, Wong $K$ and

Kumar D (2021) Differences in Levodopa Response for Progressive and Non-Progressive Micrographia in

Parkinson's Disease.

Front. Neurol. 12:665112. doi: 10.3389/fneur.2021.665112
Background: Micrographia, one element of the dysgraphia of Parkinson's disease (PD), may be classified according to the presence or absence of a decremental pattern. The decremental form, progressive micrographia, is an expression of the sequence effect seen generally in bradykinesia. Its responsiveness to levodopa has not been evaluated kinematically.

Objectives: Aim of this study is to investigate the difference in levodopa response for progressive and non-progressive micrographia.

Methods: Twenty-four PD patients and 24 age-matched repeatedly wrote the letter e on a computerized digital tablet. PD patients performed the task two times, in a defined off state and again after levodopa. Scripts were classified as progressive micrographia (PDPM) or non-progressive micrographia (PDNPM) depending on whether a $10 \%$ decrement was seen between the first and final characters of a line of lettering.

Results: While levodopa produced a similar response on the MDS-UPDRS motor scale for the two groups, the effect on the two types of micrographia was different. While writing speed improved significantly in both groups after levodopa, the responses were over twofold greater for PD $D_{\mathrm{NPM}}$. Moreover, the decremental features of $\mathrm{PD}_{\mathrm{PM}}$-in size, speed, and pen-pressure - were largely unaltered by a levodopa dose.

Conclusions: Progressive micrographia is less responsive to levodopa. Our findings agree with research showing that the sequence effect of bradykinesia is relatively resistant to medication. Yet we did not find a weaker overall levodopa motor benefit. Caution is needed in the interpretation of such micrographia measurements for estimating drug responses.

Keywords: Parkinson's disease, levodopa, kinematic, micrographia, dysgraphia

\section{INTRODUCTION}

Impairment of handwriting is commonly present in Parkinson's disease (PD), and may predate other symptoms (1). Although reduced size is the most recognized feature, the use of computerized graphic tablets shows that parkinsonian dysgraphia comprises defects in amplitude, velocity, and fluency (2-4). Kinnear Wilson observed that the script size of parkinsonian subjects often trailed 
off as they continued to write. He proposed a subdivision into progressive micrographia, where there was decrement of letter size across a line of text, and consistent micrographia, where the size of letters was evenly reduced (5).

Other authors have used this classification $(6,7)$. Wu et al. (8) found that the two types of parkinsonian micrographia show different patterns of activation of the motor system on functional magnetic resonance scans. There are, however, difficulties with definitions of consistent micrographia. Kinnear Wilson suggested a comparison with pre-morbid calligraphy. The establishment of a 'typical' historical script size is one limitation, since writing size varies considerably in a normal subject depending on writing context (9). In a previous study, we found that classifications of consistent micrographia based on letter size did not yield a distinct subset (10). Only $4 \%$ had a purely consistent pattern, while some PD patients with decrement also fell below the consistent cut-off. We concluded that parkinsonian dysgraphia is better classified according to whether progressive micrographia, defined by a $10 \%$ decrement between first five and last five sets of sequential letters, is present $\left(\mathrm{PD}_{\mathrm{PM}}\right)$ or absent $\left(\mathrm{PD}_{\mathrm{NPM}}\right)$.

Progressive micrographia is an expression of the sequence effect of bradykinesia-progressive reduction in speed and amplitude of repetitive actions. The Queen Square Brain Bank criteria sets a decremental description of bradykinesia at the core of the clinical diagnosis of PD (11). Interestingly, while letter size in progressive supranuclear palsy is smaller than in $\mathrm{PD}$, the decrement effect is less conspicuous (12), suggesting that non-progressive micrographia is more typical of progressive supranuclear palsy.

Levodopa has mixed effects on parkinsonian dysgraphia. Two studies that identified groups with progressive micrographia found that decrement in size was relatively unresponsive to levodopa, although neither examined kinematic aspects of writing $(6,7)$. Handwriting, by virtue of its overlearned, repetitive nature, lends itself to a kinematic examination of the elusive nature of bradykinesia and the effect of dopaminergic treatment on it $(13,14)$. While objective handwriting analysis has been suggested as a marker for early detection of PD and for monitoring of subsequent progression, previous research is divided on specific features of the levodopa response.

The aim of this work was to investigate the effect of levodopa on dimensional and kinematic measurements of handwriting in participants with and without progressive micrographia. A control group was included to allow comparison with normal left-to-right writing characteristics.

\section{MATERIALS AND METHODS}

\section{Participants}

Twenty-four patients diagnosed with PD within the last 10 years were recruited from the Movement Disorders Clinic at Monash Medical Centre. All complied with the Queen Square Brain Bank criteria for idiopathic PD (11). Twenty-four healthy agematched controls were also recruited as normal writing controls. The presence of any advanced PD clinical milestone-visual hallucinations, frequent falling, cognitive disability, or need for institutional care-was an exclusion criterion (15). Cognition was assessed for all participants using the Montreal Cognitive Assessment (MoCA) (16).

For PD participants, the writing tasks were first performed in a practically defined off state (fasting, with anti-parkinsonian medication withheld for at least $12 \mathrm{~h}$ ) (17). Subjects' usual morning levodopa dose was then administered, their on state taken as the maximum improvement over the next 30$90 \mathrm{~min}$. Motor function in off and on states was scored by a neurologist on the Movement Disorders Society Unified Parkinson's Disease Rating Scale Part III (MDS-UPDRS-III) (18). Scoring of items 3.4-3.8 was extracted as a measure of dominant upper limb bradykinesia. All PD participants were taking levodopa-containing medication. Seven were using extended release levodopa. Levodopa equivalent daily doses were calculated using standard conversion factors (19). Demographic details, including handedness, of PD and control groups are shown in Tables 1, 2. Table 3 presents clinical information on individual PD subjects. The study was conducted in accordance with the Helsinki Declaration on human experiments (revised 2004) and was approved by the Monash Health and RMIT University Human Research Ethics Committees. All participants in this study gave their written informed consent prior to data recording.

\section{Data Recording Methods}

A digital tablet (Wacom Intuos Pro-Large) with the ink-pen having a pressure sensor was used for the experiments. The advantage of this device was that it gave participants the feeling of conventional pen and paper and was likely to be less defamiliarizing to older subjects. Participants sat on a chair, and the tablet was placed on an adjustable desk. The table height and positioning of the tablet on the table was adjusted according to participant preference.

Customized software was developed to record and analyse data from the tablet. The software registered the $x-y$ coordinates, pen pressure and time stamp at a $133 \mathrm{~Hz}$ sampling rate.

\section{Handwriting Tasks and Computation of Handwriting Features}

The task of repeatedly writing of the letter e is routinely used by clinicians to test for micrographia. In this study, the size of each letter e was obtained using $\mathrm{x}$ and $\mathrm{y}$ coordinates (in $\mathrm{mm}$ ) and Euclidean formula to calculate stroke length (10). Participants were instructed to write the letter e repeatedly, with pen-up at the end of each letter (see Figure 1). Once 20 repetitions had been exceeded, a researcher gave the instruction to stop writing. Similar protocols have previously been used to study micrographia $(10,20,21)$.

The writing data was first segmented based on pen-up and pen-down motions. A threshold of $5 \mathrm{~mm}$ was used, and segments of length less than the threshold were excluded. Speed was calculated using a weighted average based on the stroke length measurement of each letter (22).

The initial and final sets of five letters were compared. Progressive micrographia was defined as $10 \%$ or greater decrement in the average size of the letter $e$ in the off state (10). PD participants who met this definition were classified as 
TABLE 1 | Demographic and clinical information, PD patients and controls.

\begin{tabular}{lccc}
\hline Demographics & PD & Control group & p value \\
\hline Number of Subjects, $n$ & 24 & 24 & \\
Age, years & $71.6 \pm 7.14$ & $69.3 \pm 5.74$ & $0.2^{a}$ \\
Gender male, female & 13,11 & 14,10 & $1.0^{b}$ \\
Handedness Right, Left & 20,4 & 22,2 & $0.7^{b}$ \\
Disease duration, years & $5.0 \pm 2.88$ & - & \\
Levodopa equivalent & $480 \pm 296$ & - & \\
daily dosage (mg) & & &
\end{tabular}

Values are mean \pm standard deviation, comparison between groups was performed using ${ }^{a}$ Independent $t$ test and ${ }^{b}$ Chi-Square 2-tailed test.

TABLE 2 | Demographic and clinical information for PDPM and PDNPM groups.

\begin{tabular}{lccc}
\hline Demographics & PD $_{\mathrm{PM}}$ & PD $_{\mathrm{NPM}}$ & $\boldsymbol{p}$ values \\
\hline Number of Subjects, $n$ & 16 & 8 & \\
Age, years & $70.94 \pm 7.59$ & $73.63 \pm 6.23$ & $0.4^{a}$ \\
Gender male, female & 10,6 & 3,5 & $0.35^{b}$ \\
Handedness Right, Left & 14,2 & 5,1 & $0.83^{b}$ \\
Disease duration, years & $5.1 \pm 2.8$ & 5.3 & $0.84^{a}$ \\
MDS-UPDRS-III OFF [0-132] & $28.53 \pm 10.33$ & $23.88 \pm 7.80$ & $0.28^{a}$ \\
MDS-UPDRS-III ON [0-132] & $20.25 \pm 9.99$ & $18.88 \pm 3.53$ & $0.73^{a}$ \\
Dominant upper limb & $3.88 \pm 1.67$ & $3.13 \pm 1.46$ & $0.29^{a}$ \\
bradykinesia score OFF & & & \\
[0-12] & & & $0.24^{a}$ \\
Dominant upper limb & $3.13 \pm 1.89$ & $2.13 \pm 1.96$ & \\
bradykinesia score ON & & & \\
[0-12] & & & \\
\hline
\end{tabular}

Values are mean \pm standard deviation except for gender and handedness. Comparisons between groups performed using ${ }^{a}$ Independent $t$-test, and ${ }^{b}$ Mann-Whitney $U$ test.

$\mathrm{PD}_{\mathrm{PM}}$, the remainder as $\mathrm{PD}_{\mathrm{NPM}}$. Figure 1 shows an example of progressive micrographia.

\section{Statistical Analysis}

Independent sample $t$-test was performed to compare PD and control groups to confirm age-matching, while Chi-Square test was performed for differences in gender and handedness (10). The demographic data was compared between groups using independent $t$-test, while Mann-Whitney $\mathrm{U}$ test was used for group differences in gender and right-handedness.

As the data was not normally distributed based on ShapiroWilk test, non-parametric Wilcoxon Signed-rank test was performed on related samples from each subject to understand the relationship between the initial and final letter series. MannWhitney $U$ test (2-tailed) is suitable for two group analysis hence selected to study the difference between the groups (23).

The sample size of 24 for PD and control groups was determined by a power calculation performed using an online power and sample size calculator developed by Statistical Solutions software (24). The parameters for this were: statistical power $=0.8$, with $95 \%$ confidence interval and assuming a mean difference between the groups of $110 \mathrm{~mm} / \mathrm{sec}^{2}$ and pooled standard deviation of 140 (10).

\section{RESULTS}

Sixteen PD participants showed a decrement $>10 \%$ when off and were categorized as $\mathrm{PD}_{\mathrm{PM}}$. Based on Wilcoxon test, initial and final sizes were significantly different $(p<0.001)$ in this group. This difference narrowed when on and was no longer significant. The remaining $8 \mathrm{PD}$ participants composed the $\mathrm{PD}_{\mathrm{NPM}}$ group. In $\mathrm{PD}_{\mathrm{NPM}}$, there was a small increase in the size of the character $\mathrm{e}$ from initial to the final, which was significant in the off but not the on state (see Figure 2). Controls showed a slight increase in letter size from initial to final.

We found that control participants exhibited an increment in the speed of writing from left to right. $\mathrm{PD}_{\mathrm{NPM}}$, though slower than controls, also increased their speed across the line of letters in their off state. With the $\mathrm{PD}_{\mathrm{PM}}$ group, there was little change in speed between initial and the final letters.

$\mathrm{PD}_{\mathrm{NPM}}$ scripts showed greater responses to levodopa than $\mathrm{PD}_{\mathrm{PM}}$, and this effect was stronger for letter speed than size. Writing speeds were significantly greater for $\mathrm{PD}_{\mathrm{NPM}}$ when on, and the increase in speed from the initial to final letters remained obvious. Smaller though significant speed increase was seen when on in the $\mathrm{PD}_{\mathrm{PM}}$ group, but without speed change from initial to final letters.

Comparisons with control data in Table 4 indicate the relative magnitude of levodopa effects. Letter size for $\mathrm{PD}_{\mathrm{NPM}}$ approached control values after levodopa, both for initial and final characters, with no significant differences. Speed, expressed in $\mathrm{mm} / \mathrm{sec}$, improved after levodopa in the $\mathrm{PD}_{\mathrm{NPM}}$ group by 7.1 (initial letters) and 10.5 (final letters). The difference between on state $\mathrm{PD}_{\mathrm{NPM}}$ and control writing speed for final letters also became insignificant $(p=0.26)$. The levodopa effect on speed in $\mathrm{PD}_{\mathrm{PM}}$ was much smaller-only 3.1 (initial letters) and 3.6 (final letters) - with persisting differences from control values $(p=0.001)$.

The $\mathrm{PD}_{\mathrm{PM}}$ group were not able to maintain pen-pressure from left to right. This produced a significant initial to final difference in the off state, with little improvement when on. Pen-pressure was more consistently applied in $\mathrm{PD}_{\mathrm{NPM}}$ scripts, for both off and on.

Tables 4, 5 demonstrate that, overall, the decremental aspects of $\mathrm{PD}_{\mathrm{PM}}$ obtained little levodopa benefit. Comparing initial and final letters, size and pen-pressure decrements remained, while the lack of left-to-right speed increment was not remedied.

As shown in Table 2, the MDS-UPDRS-III scale registered a significant response to levodopa $(p<0.05)$ for both groups of PD patients. The $\mathrm{PD}_{\mathrm{NPM}}$ group improved from 23.9 to 18.9 , an overall motor response score of 5.0, while for PDPM the improvement was 8.2.

Table 4 displays the size and speed of writing of the character e for $\mathrm{PD}_{\mathrm{PM}}$ and $\mathrm{PD}_{\mathrm{NPM}}$ groups in off and on states, along with the control values. This data is embedded in Figures 2, 3. The group difference statistical analysis performed using Mann-Whitney tests is shown in Table 5.

\section{DISCUSSION}

Levodopa had significantly different effects on parkinsonian dysgraphia depending on whether or not progressive 
TABLE 3 | Clinical information on PD participants.

\begin{tabular}{|c|c|c|c|c|c|c|c|}
\hline & Age & Gender & $\begin{array}{l}\text { PD duration } \\
\text { (years) }\end{array}$ & $\begin{array}{l}\text { levodopa equivalent dose } \\
\text { (mg) }\end{array}$ & $\begin{array}{l}\text { MDS-UPDRS-III } \\
\text { off }\end{array}$ & $\begin{array}{l}\text { MDS-UPDRS-III } \\
\text { on }\end{array}$ & Micrographia classification \\
\hline 1 & 81 & $\mathrm{~F}$ & 8 & 600 & 23 & 15 & $P D_{N P M}$ \\
\hline 3 & 62 & M & 6 & 950 & 47 & 29 & PDPM \\
\hline 4 & 82 & M & 7 & 750 & 27 & 22 & PDPM \\
\hline 7 & 70 & M & 5 & 600 & 25 & 20 & PDPM \\
\hline 8 & 70 & M & 1 & 500 & 15 & 10 & $P D_{N P M}$ \\
\hline 9 & 76 & M & 3 & 150 & 25 & 15 & $P D_{P M}$ \\
\hline 10 & 70 & $\mathrm{~F}$ & 1.5 & 300 & 43 & 40 & PDPM \\
\hline 11 & 66 & M & 5 & 300 & 40 & 37 & $P D_{N P M}$ \\
\hline 16 & 64 & $\mathrm{~F}$ & 5 & 300 & 12 & 12 & $\mathrm{PD}_{\mathrm{PM}}$ \\
\hline 17 & 64 & $\mathrm{~F}$ & 6 & 300 & 8 & 4 & PDPM \\
\hline 18 & 72 & $M$ & 9 & 950 & 33 & 12 & PDPM \\
\hline 19 & 66 & $\mathrm{~F}$ & 10 & 620 & 30 & 20 & PDPM \\
\hline 20 & 77 & F & 8 & 300 & 15 & 11 & $P D_{N P M}$ \\
\hline 21 & 75 & $M$ & 1.5 & 300 & 25 & 24 & $P D_{N P M}$ \\
\hline 22 & 78 & M & 8 & 450 & 25 & 21 & PDPM \\
\hline 23 & 83 & F & 1.5 & 300 & 26 & 18 & PDPM \\
\hline 24 & 77 & $\mathrm{~F}$ & 10 & 1500 & 22 & 14 & $P D_{N P M}$ \\
\hline
\end{tabular}

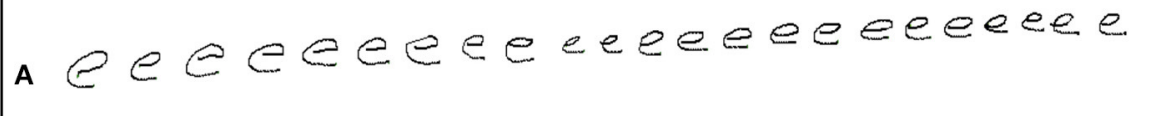

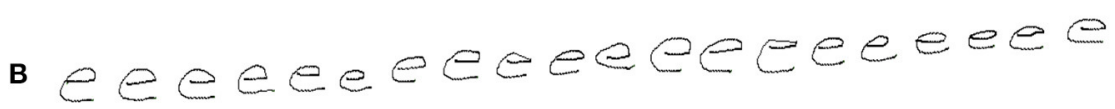

FIGURE 1 | An example of repetition of the letter e by a PD participant with progressive micrographia: off state (A) and on state (B).

micrographia is present. PDPM had less levodopa improvement to writing in general. Notably, the decremental features of progressive micrographia are relatively resistant to dopaminergic treatment. One observation about normal handwriting is important in understanding our findings. The control group showed an increase in speed from initial to final letters. This possibly reflects changes in muscle activation as wrist and elbow movement come increasingly into play when writing from left to right (25). The same tendency was present in $\mathrm{PD}_{\mathrm{NPM}}$ (10). $\mathrm{PD}_{\mathrm{PM}}$ lacks this left-to-right augmentation of writing speed.

The $\mathrm{PD}_{\mathrm{NPM}}$ group had increases in both letter size and writing speed after levodopa that were greater than those seen in PDPM.
In $\mathrm{PD}_{\mathrm{NPM}}$ the greatest improvements in on state writing speed were seen in the final letter series, approaching the values of control left-to-right speed augmentation, and showing strong statistical significance. In $\mathrm{PD}_{\mathrm{PM}}$, improvement in speed after levodopa was only half as much, and the blunting of left-toright augmentation of writing speed largely persisted. The other aspect of motor decrement in $\mathrm{PD}_{\mathrm{PM}}$, a fall-off in pen pressure with final letters, was similar before and after levodopa. Figure 1 provides a good illustration of the findings in $\mathrm{PD}_{\mathrm{PM}}$. Some improvement in overall letter size is present after levodopa in this specimen, including the final characters. But waves of decrement are still obvious. 


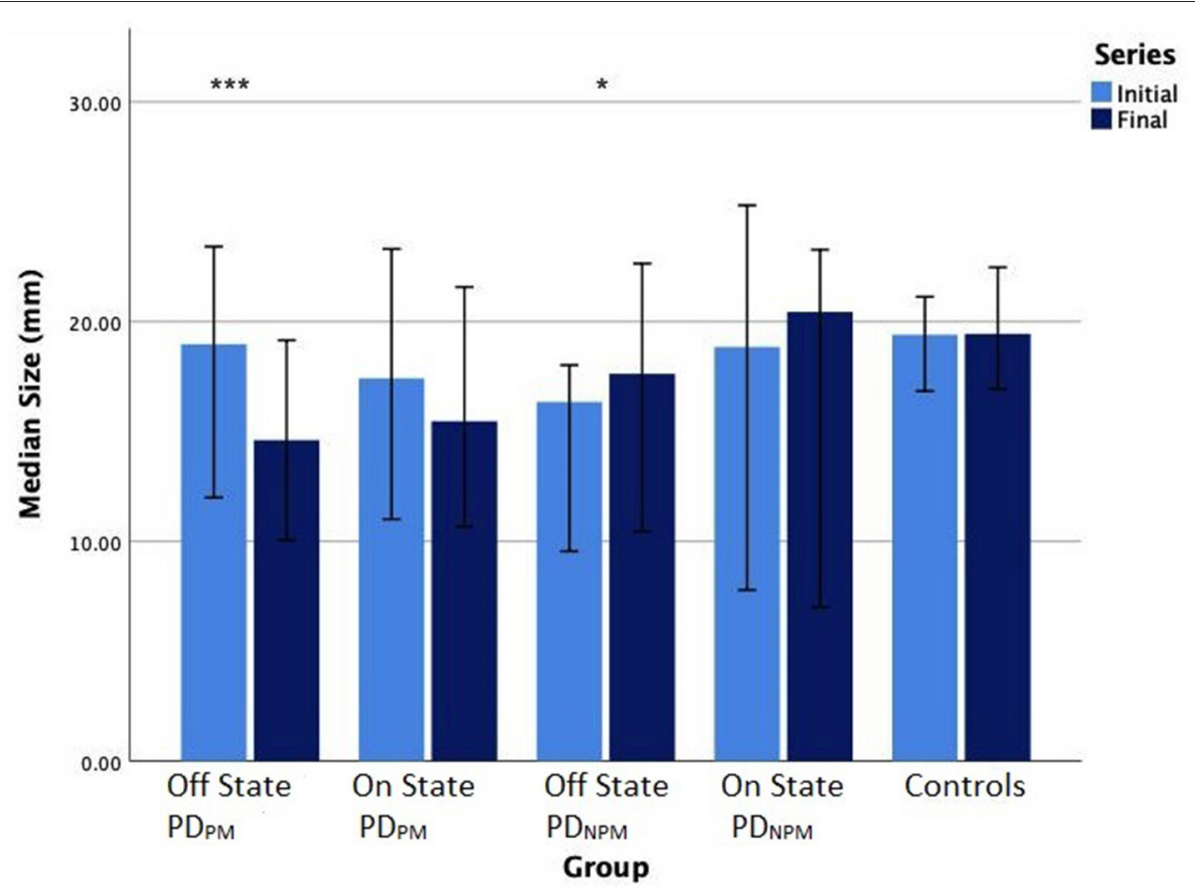

FIGURE 2 | Bar chart (error bars for 95\% confidence intervals) of median writing size (mm) of PD participants in off and on states, and controls. Wilcoxon signed-rank test significance levels: ${ }^{* \star *} P<0.001,{ }^{*} P<0.05$.

TABLE 4 | Size and speed of character writing, and pen-pressure, for different groups of PD and controls.

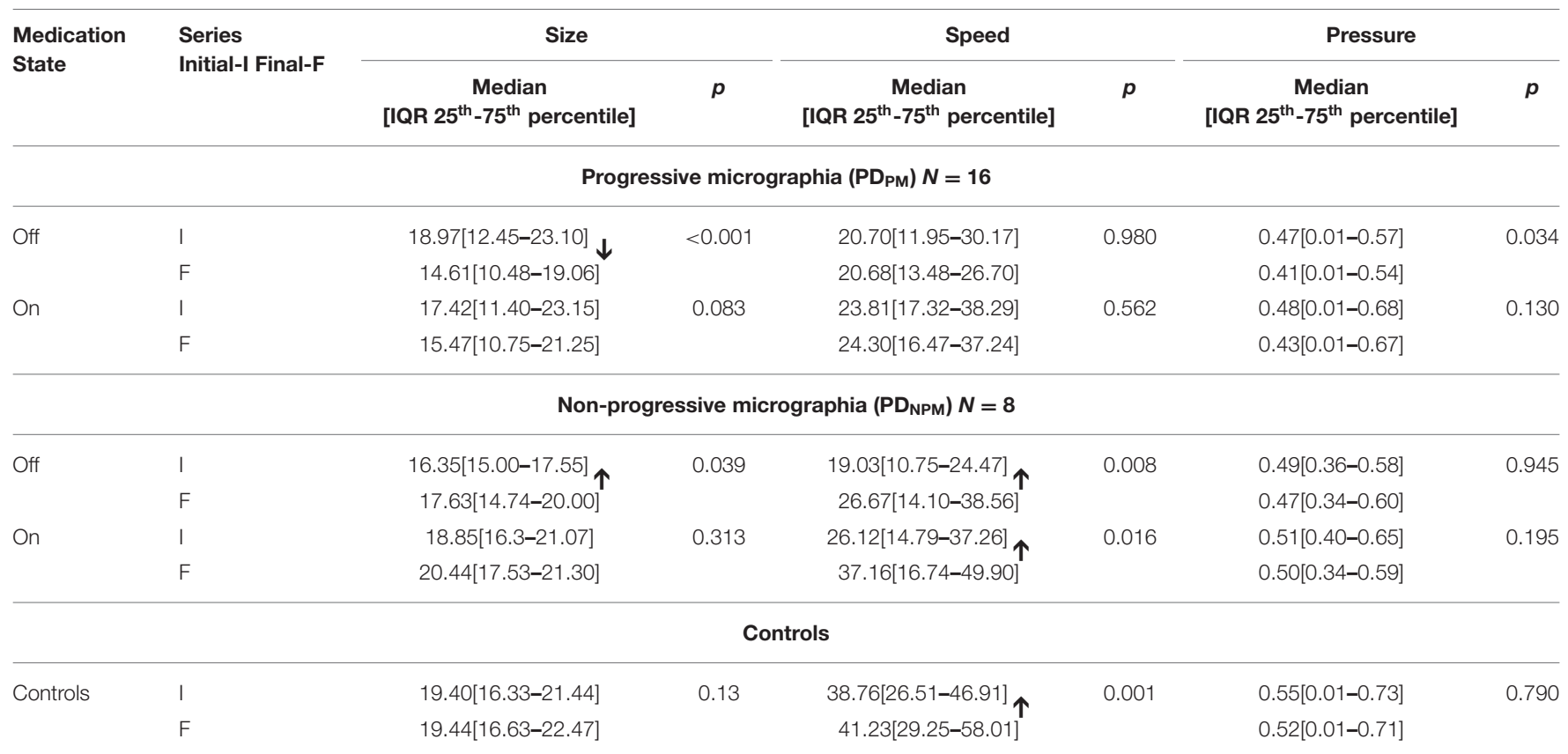

Two-tailed Wilcoxon signed-rank test was performed to compare initial and final series. IQR, interquartile range.

Our finding that the writing decrements of $\mathrm{PD}_{\mathrm{PM}}$ show little responsiveness to levodopa broadly agrees with some previous research. Ling et al. (8) and $\mathrm{Wu}$ et al. (12) each used different definitions of progressive micrographia, and neither conducted kinematic measurements. Both reported that decrements in script size persisted in patients with PD despite levodopa therapy. Ling et al. (12) found a modest improvement in writing size in the on phases of their subjects. In $\mathrm{Wu}$ et al. (8) study, levodopa hardly changed character size when progressive micrographia was present, whereas there was significant improvement in a consistent micrographia group. 
TABLE 5 | Mann-Whitney test ${ }^{\mathrm{a}}$ and Wilcoxon test $\mathrm{t}^{\mathrm{b}} p$-values for group differences, including medication effects.

\begin{tabular}{|c|c|c|c|c|c|c|}
\hline Groups & Speed (Initial) & Speed (Final) & Size (Initial) & Size (Final) & Pen-pressure (Initial) & Pen-pressure (Final) \\
\hline $\mathrm{PD}_{\mathrm{NPM}}-\mathrm{Off}$ and Controls ${ }^{\mathrm{a}}$ & 0.001 & 0.041 & 0.029 & 0.258 & 0.535 & 0.728 \\
\hline $\mathrm{PD}_{\mathrm{NPM}}-\mathrm{On}$ and Controls ${ }^{\mathrm{a}}$ & 0.041 & 0.258 & 0.815 & 0.931 & 0.815 & 0.896 \\
\hline PDPM-Off and Controls ${ }^{a}$ & 0.001 & $<0.001$ & 0.890 & 0.009 & 0.456 & 0.307 \\
\hline PDPM-On and Controls ${ }^{a}$ & 0.016 & 0.001 & 0.508 & 0.064 & 0.804 & 0.699 \\
\hline PDPM-Off and PDPM-On ${ }^{b}$ & 0.006 & 0.011 & 0.234 & 0.255 & 0.159 & 0.464 \\
\hline $\mathrm{PD}_{\mathrm{NPM}}-\mathrm{Off}$ and $\mathrm{PD}_{\mathrm{NPM}}-\mathrm{On}^{\mathrm{b}}$ & 0.017 & 0.069 & 0.069 & 0.208 & 0.208 & 0.401 \\
\hline
\end{tabular}

Significant $p$-values shown in bold type.

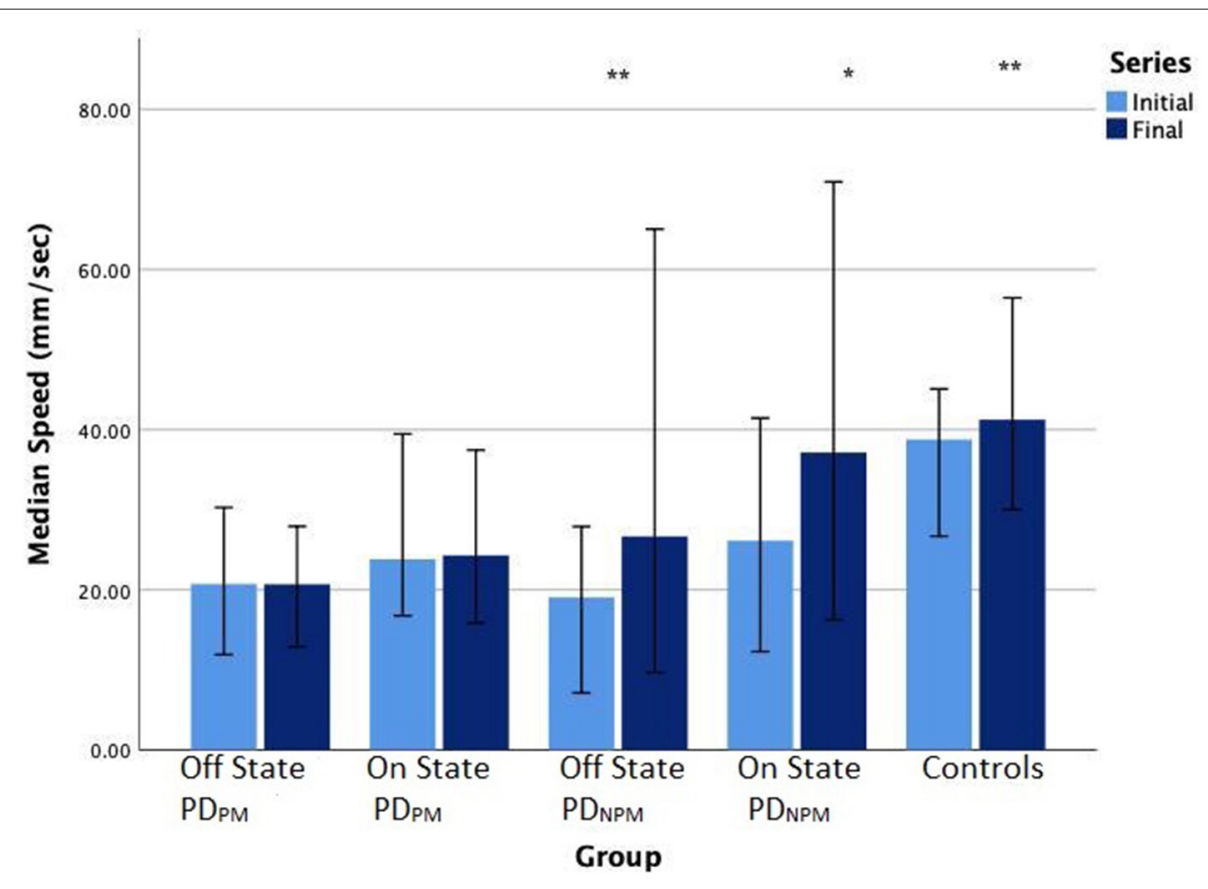

FIGURE 3 | Bar chart (error bars for 95\% confidence intervals) of median writing speeds (mm/sec) of PD participants in off and on states, and controls. Wilcoxon signed-rank test significance levels: ${ }^{\star \star} P<0.01,{ }^{\star} P<0.05$.

While we found progressive micrographia is generally less responsive to levodopa, this did not correspond to overall parkinsonian motor disability or its responsiveness to levodopa. The PDPM group had a marginally higher MDS-UPDRS-III score. The motor response to levodopa was, however, a little better for $\mathrm{PD}_{\mathrm{PM}}$ with an improvement of 8.0 compared with 5.3 for $\mathrm{PD}_{\mathrm{NPM}}$. Dominant upper limb bradykinesia was slightly greater for $\mathrm{PD}_{\mathrm{PM}}$, with a slightly smaller levodopa response. None of these differences were significant. Wu et al. (8) had also shown that progressive micrographia is not associated with a worse overall motor disability score, though comparisons of levodopa motor benefit were not given.

Bradykinesia is a shorthand for complex disturbances of initiation and execution of actions and the ability to sustain them. Slowness is not necessarily its most prominent component. Akinesia (failure to initiate movement) and hypokinesia (underactive movement) are part of bradykinesia, as is the sequence effect-repetitive movements becoming smaller or slower. Handwriting analysis has shown reduced speed and size in $\mathrm{PD}$, and this has been used for the detection and monitoring of disease symptoms $(22,26,27)$. The defining feature of progressive micrographia, though, represents the sequence effect. Research into other manifestations of the sequence effect mirrors our observations in handwriting. Kang et al. (28) using a pegboard task, found that levodopa improved overall speed but not decrement. Several studies have looked at bradykinesia in finger tapping $(12,29,30)$. While a range of kinematic speed and rhythm measures are improved with levodopa, there is little change in decrement of amplitude.

Using abstract computerized methods, Gangadhar et al. (31) modeled progressive micrographia as a specific combination of moment-to-moment depletion of striatal dopamine and basal ganglia network dynamics. The sequence effect appears to be an integral component of bradykinesia. Parkinsonian movements are generally underscaled with respect to desirable range and speed, consistent with a defect in 'motor energy'. 
Decrement, therefore, seems to reflect cumulative energy cost, (32) perhaps explaining why it has a poorer dopaminergic response than other kinematic or dimensional aspects of writing.

This study has shown some caveats on the use of handwriting analysis to monitor the dopaminergic responses of patients with PD. There are two patterns of dysgraphia in $\mathrm{PD}$-one with progressive micrographia and one without. The significant differences in their handwriting response to levodopa appear unrelated to the overall motor benefit from the drug. While we are unable to conclude the mechanisms underpinning these differences, we recommend that the two should be distinguished in handwriting research because of their dissimilar pharmacological characteristics. Further studies need to investigate the energy costs of progressive micrographia. One possible explanation is that people with this form of parkinsonian dysgraphia are more impacted by sequence effects than those without.

\section{LIMITATIONS}

The study sample size used here is similar to otherwise comparable studies, though not sufficient to fully investigate possible demographic factors. A defined off state and test-dose method is a standard way to evaluate the levodopa motor response. This method did, however, impose an unvarying order of off and on measurements. Another limitation is that effect of dosage was not assessed and hence it is not possible to comment on treatment options.

\section{CONCLUSION}

The effect of levodopa on parkinsonian dysgraphia is reduced by the presence of progressive micrographia. The decrement of progressive micrographia is a manifestation of the sequence effect, the aspect of bradykinesia that is most resistant to dopaminergic treatment. Nevertheless, it does not denote that general motor disability is greater, or shows less response to levodopa. Computerized handwriting analysis has been proposed as a way of tracking the progression of PD and efficacy

\section{REFERENCES}

1. McLennan JE, Nakano K, Tyler HR, Schwab RS. Micrographia in Parkinson's disease. J Neurol Sci. (1972) 15:141-52. doi: 10.1016/0022-510X(72)90002-0

2. Rosenblum S, Samuel M, Zlotnik S, Erikh I, Schlesinger I. Handwriting as an objective tool for Parkinson's disease diagnosis. J Neurol. (2013) 260:2357-61. doi: 10.1007/s00415-013-6996-x

3. Smits EJ, Tolonen AJ, Cluitmans L, van Gils M, Conway BA, Zietsma RC, et al. Standardized handwriting to assess bradykinesia, micrographia and tremor in Parkinson's disease. PLoS ONE. (2014) 9:e97614. doi: 10.1371/journal.pone.0097614

4. Letanneux A, Danna J, Velay JL, Viallet F, Pinto S. From micrographia to Parkinson's disease dysgraphia. Mov Disord. (2014) 29:1467-75. doi: $10.1002 / \mathrm{mds} .25990$

5. Wilson K. Croonian lectures. Lancet. (1925) 2:53. of antiparkinsonian treatment. By highlighting relationships between progressive micrographia and medication response, our findings imply that caution is needed in the interpretation of such measurements.

\section{DATA AVAILABILITY STATEMENT}

The raw data supporting the conclusions of this article will be made available by the authors, without undue reservation.

\section{ETHICS STATEMENT}

The studies involving human participants were reviewed and approved by the study was conducted in accordance with the Helsinki Declaration on human experiments (revised 2004) and was approved by the Monash Health and RMIT University Human Research Ethics Committees. All participants in this study gave their written informed consent prior to data recording. The patients/participants provided their written informed consent to participate in this study.

\section{AUTHOR CONTRIBUTIONS}

PZ involved in conducting experiments, data analysis, drafting the article, software design and development, selection of analytical tools, statistical analysis, and literature review. DK involved in concept and design of work, selection of analytical tools, critical revision of the article, literature review, participated in manuscript preparation, and final approval of the version to be published. PK involved in clinical support, critical revision of the article, and participated in manuscript preparation. SP involved in statistical analysis, and review of the article. SR involved in clinical support, KW and KN involved in experimental support. All authors were involved in manuscript review.

\section{FUNDING}

We acknowledge the funding supported by RMIT University scholarship and clinical support from Monash Medical Center, Melbourne, Australia. 
11. Hughes AJ, Daniel SE, Kilford L, Lees AJ. Accuracy of clinical diagnosis of idiopathic Parkinson's disease: a clinico-pathological study of 100 cases. J Neurol Neurosurg Psychiatry. (1992) 55:181-4. doi: 10.1136/jnnp.55.3.181

12. Ling H, Massey LA, Lees AJ, Brown P, Day BL. Hypokinesia without decrement distinguishes progressive supranuclear palsy from Parkinson's disease. Brain. (2012) 135:1141-53. doi: 10.1093/brain/aws038

13. Drotár P, Mekyska J, Smékal Z, Rektorová I, Masarová L, Faundez-Zanuy M. Prediction potential of different handwriting tasks for diagnosis of parkinson's. In 2013 E-Health and Bioengineering Conference (EHB). Iasi: IEEE (2013). p. 1-4. doi: 10.1109/EHB.2013.6707378

14. Pereira CR, Pereira DR, da Silva FA, Hook C, Weber SAT, Pereira LAM, et al. A step towards the automated diagnosis of parkinson's disease: analyzing handwriting movements. In 2015 IEEE 28th International Symposium on Computer-Based Medical Systems. Sao Carlos: IEEE (2015). p. 171-6.

15. Kempster PA, O'Sullivan SS, Holton JL, Revesz T, Lees AJ. Relationships between age and late progression of Parkinson's disease: a clinico-pathological study. Brain. (2010) 133:1755-62. doi: 10.1093/brain/awq059

16. Nasreddine ZS, Phillips NA, Bédirian V, Charbonneau S, Whitehead V, Collin I, et al. The Montreal Cognitive Assessment, MoCA: a brief screening tool for mild cognitive impairment. J Am Geriatr Soc. (2005) 53:695-9. doi: 10.1111/j.1532-5415.2005.53221.x

17. Defer GL, Widner H, Marié RM, Rémy P, Levivier M. Core assessment program for surgical interventional therapies in Parkinson's disease (CAPSITPD). Mov Disord. (1999) 14:572-84.

18. Goetz CG, Tilley BC, Shaftman SR, Stebbins GT, Fahn S, Martinez-Martin P, et al. Movement disorder society-sponsored revision of the unified Parkinson's disease rating scale (MDS-UPDRS): scale presentation and clinimetric testing results. Mov Disord. (2008) 23:2129-70. doi: 10.1002/mds.22340

19. Tomlinson CL, Stowe R, Patel S, Rick C, Gray R, Clarke CE. Systematic review of levodopa dose equivalency reporting in Parkinson's disease. Mov Disord. (2010) 25:2649-53. doi: 10.1002/mds.23429

20. Teulings H-L, Stelmach GE. Control of stroke size, peak acceleration, and stroke duration in Parkinsonian handwriting. Hum Mov Sci. (1991) 10:31534. doi: 10.1016/0167-9457(91)90010-U

21. Thomas M, Lenka A, Kumar Pal P. Handwriting Analysis in Parkinson's disease: current status and future directions. Mov Disord Clin Pract. (2017) 4:806-18. doi: $10.1002 / \mathrm{mdc} 3.12552$

22. Zham P, Kumar DK, Dabnichki P, Arjunan S, Raghav S. Distinguishing different stages of Parkinson's disease using composite index of speed and pen-pressure of sketching a spiral. Front Neurol. (2017) 8:435. doi: 10.3389/fneur.2017.00435

23. du Prel J-B, Röhrig B, Hommel G, Blettner M. Choosing statistical tests: part 12 of a series on evaluation of scientific publications.
Dtsch Arztebl Int. (2010) 107:343. doi: 10.3238/arztebl.201 0.0343

24. Rosner B. Fundamentals of Biostatistics. xvii, 859 p. Boston, MA: Brooks/Cole, Cengage Learn Bost (2011). Available online at: https://clincalc.com/Stats/ SampleSize.aspx

25. Kushki A, Schwellnus H, Ilyas F, Chau T. Changes in kinetics and kinematics of handwriting during a prolonged writing task in children with and without dysgraphia. Res Dev Disabil. (2011) 32:1058-64. doi: 10.1016/j.ridd.2011.01.026

26. Drotár P, Mekyska J, Rektorová I, Masarová L, Smékal Z, Faundez-Zanuy M. Evaluation of handwriting kinematics and pressure for differential diagnosis of Parkinson's disease. Artif Intell Med. (2016) 67:39-46. doi: 10.1016/j.artmed.2016.01.004

27. San Luciano M, Wang C, Ortega RA, Yu Q, Boschung S, Soto-Valencia J, et al. Digitized Spiral Drawing: A Possible Biomarker for Early Parkinson's Disease. PLoS ONE. (2016) 11:e0162799. doi: 10.1371/journal.pone.0162799

28. Kang SY, Wasaka T, Shamim EA, Auh S, Ueki Y, Lopez GJ, et al. Characteristics of the sequence effect in Parkinson's disease. Mov Disord. (2010) 25:2148-55. doi: $10.1002 / \mathrm{mds} .23251$

29. Bologna M, Guerra A, Paparella G, Giordo L, Fegatelli DA, Vestri AR, et al. Neurophysiological correlates of bradykinesia in Parkinson's disease. Brain. (2018) 141:2432-44. doi: 10.1093/brain/awy155

30. Bologna M, Paparella G, Fasano A, Hallett M, Berardelli A. Evolving concepts on bradykinesia. Brain. (2020) 143:727-50. doi: 10.1093/brain/awz344

31. Gangadhar G, Joseph D, Chakravarthy VS. Understanding parkinsonian handwriting through a computational model of basal ganglia. Neural Comput. (2008) 20:2491-525. doi: 10.1162/neco.2008.03-07-498

32. Tinaz S, Lauro P, Hallett M, Horovitz SG. Deficits in task-set maintenance and execution networks in Parkinson's disease. Brain Struct Funct. (2016) 221:1413-25. doi: 10.1007/s00429-014-0981-8

Conflict of Interest: PZ was employed by the company IBM Research Australia.

The remaining authors declare that the research was conducted in the absence of any commercial or financial relationships that could be construed as a potential conflict of interest.

Copyright (c) 2021 Zham, Poosapadi, Kempster, Raghav, Nagao, Wong and Kumar. This is an open-access article distributed under the terms of the Creative Commons Attribution License (CC BY). The use, distribution or reproduction in other forums is permitted, provided the original author(s) and the copyright owner(s) are credited and that the original publication in this journal is cited, in accordance with accepted academic practice. No use, distribution or reproduction is permitted which does not comply with these terms. 